\title{
Robust Consensus for a Class of Uncertain Multi-Agent Dynamical Systems
}

\author{
Dongkun Han, Student Member, IEEE, Graziano Chesi, Senior Member, IEEE, \\ and Yeung Sam Hung, Senior Member, IEEE
}

\begin{abstract}
This paper investigates robust consensus for a class of uncertain multi-agent dynamical systems. Specifically, it is supposed that the system is described by a weighted adjacency matrix whose entries are polynomial functions of an uncertain vector constrained in a semialgebraic set. For this uncertain topology, we provide necessary and sufficient conditions for ensuring robust first-order consensus and robust second-order consensus, in both cases of positive and non-positive weighted adjacency matrices. Moreover, we show how these conditions can be investigated through convex programming by using standard software. Some numerical examples illustrate the proposed results.
\end{abstract}

Index Terms-Multi-agent system, Uncertain system, Robust consensus, Convex programming.

\section{INTRODUCTION}

The model of multi-agent dynamical systems has been widely applied in the research of sensor networks, neural networks and biological networks [1]-[5]. Especially, in recent years, interests are intensively casted on networked control and coordinated behavior in multi-agent systems [6]-[12]. Achieving consensus is a key problem in this area and as a growing number of applications of multi-agent system emerges, the research on consensus gains an essential importance on various areas such as complex dynamical network, filter design for multiple sensors, synchronization, formation and rendezvous.

Traditional research topics focus on the deterministic system to establish static model, while a growing number of researches cast attentions on the uncertainties of multi-agent system according to the unexpected link failure, communication delay, interaction limit and noise interference in system [13]-[15]. A simple but compelling mathematic description of a group of autonomous agents is the Vicsek model where possible changing of the nearest neighbor sets over time is an inherent property. This model is applied to the interaction with directional information exchange, hence introducing a more general model where each edge of a weighting matrix has a positive weighting factor.

In this paper, we investigate robust consensus for uncertain multi-agent dynamical systems. In particular, it is supposed that the weighted adjacency matrix of the closed-loop system is affected by uncertain parameters, reflecting for instance

Manuscript received April 20, 2011. Accepted for publication June 9, 2012 Copyright (C) 2009 IEEE. Personal use of this material is permitted. However, permission to use this material for any other purposes must be obtained from the IEEE by sending a request to pubs-permissions@ieee.org

All authors are with the Department of Electrical and Electronic Engineering, University of Hong Kong, Pokfulam Road, Hong Kong (email:dkhan@eee.hku.hk) missing information on the control gains. Each entry of the weighted adjacency matrix is allowed to be a generic polynomial function of an uncertain vector constrained in a semialgebraic set. This framework includes typical cases such as affine linear dependence of the system coefficients on an uncertain vector constrained in a polytope. For this uncertain topology, we provide necessary and sufficient conditions for ensuring robust first-order consensus and robust second-order consensus, in both cases of positive and non-positive weighted adjacency matrices. These conditions are obtained in general by exploiting the uncertain Laplacian matrices of the system and by introducing parameter-dependent Lyapunov functions for a suitably transformed system. Moreover, we show how these conditions can be investigated through convex programming by using standard software. Some numerical examples illustrate the proposed results.

The paper is organized as follows. Section II provides the problem formulation and some preliminaries. Section III describes the proposed conditions for robust first-order consensus and robust second-order consensus. Section IV illustrates the proposed results with some numerical examples. Lastly, Section V concludes the paper with some final remarks.

\section{Preliminaries}

\section{A. Problem Formulation}

Notation:

- $\mathbb{N}, \mathbb{R}$ : natural and real number sets;

- $A^{\prime}$ : transpose of $A$;

- $A>0(A \geq 0)$ : symmetric positive definite (semidefinite) matrix $A$;

- $0_{n}$ : origin of $\mathbb{R}^{n}$;

- $1_{n}: n \times 1$ vector with all the entries equal to 1 ;

- $I$ : identity matrix (of size defined by the context);

- $\operatorname{img}(A)$ : image of matrix $A$;

- $\operatorname{ker}(A)$ : null space of matrix $A$;

- $A \otimes B$ : Kronecker product of matrices $A$ and $B$;

- $\operatorname{spc}(A)$ : set of eigenvalues of $\mathrm{A} \in \mathbb{R}^{n \times n}$, i.e.

$$
\operatorname{spc}(A)=\{\lambda \in \mathbb{C}: \operatorname{det}(\lambda I-A)=0\} .
$$

Let $\mathscr{G}=(\mathscr{A}, \mathscr{E}, G)$ be a weighted digraph of order $n$ with the set of nodes $\mathscr{A}=\left\{A_{1}, \ldots, A_{n}\right\}$, set of directed edges $\mathscr{E}$ belonging to $\mathscr{A} \times \mathscr{A}$ and a weighted adjacency matrix $G=$ $\left(G_{i j}\right)_{n \times n}$. If an information can be transmitted from the $j$-th node to the $i$-th node, a directed edge $e_{i j} \in \mathscr{E}$ is denoted, i.e. a directed edge $e_{i j} \in \mathscr{E}$ if and only if $G_{i j} \neq 0$. In particular, 
$G$ is called positive if $G_{i j}>0$ for all $i, j$, otherwise $G$ is called non-positive.

For distinct nodes $A_{i k}, k=1, \ldots, l$, let a sequence of edges $\left(A_{i}, A_{i 1}\right),\left(A_{i 1}, A_{i 2}\right), \ldots,\left(A_{i l}, A_{j}\right)$ be a directed path from $A_{i}$ to $A_{j}$. If there is a directed path between any pair of distinct notes $A_{i}$ and $A_{j}$ for graph $\mathscr{G}$, then it is denoted as a strongly connected graph. Provided that for some node $i$, there is a directed path from $i$ to any other node, the node $i$ is called a root of the graph. A directed tree is a direct graph $\mathscr{G}$ with the property that there is exactly one root and except the root, every node in $\mathscr{G}$ has exactly one parent. For a directed graph of order $n$, a spanning tree of a directed graph is a directed tree with $n-1$ edges which connect all the $n$ nodes of the graph. If any subset of edges contains or forms a spanning tree, we say that the graph has a spanning tree.

In this paper we investigate robustness of consensus to uncertain parameters. In particular, it is supposed that the weighted adjacency matrix of the closed-loop system is affected by uncertain parameters, reflecting for instance missing information on the control gains. We denote such a matrix as $G(\theta)$ where $\theta \in \mathbb{R}^{r}$ is an uncertain vector constrained as

$$
\theta \in \Omega
$$

where

$$
\Omega=\left\{\theta \in \mathbb{R}^{r}: s_{i}(\theta) \geq 0 \forall i=1, \ldots, h\right\}
$$

for some functions $s_{1}, \ldots, s_{h}: \mathbb{R}^{r} \rightarrow \mathbb{R}$. In the sequel we will assume that the entries of $G(\theta)$ and $s_{1}(\theta), \ldots, s_{h}(\theta)$ are polynomials. Moreover, we say that $G(\theta)$ is positive if $G_{i j}(\theta)>0$ for all $i, j$ and for all $\theta \in \Omega$, otherwise $G(\theta)$ is called non-positive.

For robust first-order consensus, we consider the continuous-time uncertain multi-agent dynamical system described by

$$
\dot{x}_{i}(t)=\sum_{j=1, j \neq i}^{n} G_{i j}(\theta)\left(x_{j}(t)-x_{i}(t)\right), \quad i=1, \ldots, n
$$

where $x_{i}$ is the state of the $i$-th node, and $G(\theta)$ is both positive and non-positive. The robust first-order consensus problem is as follows.

Problem 1. To establish if, for any initial state, the uncertain multi-agent dynamical system (3) achieves robust first-order consensus, i.e.

$$
\lim _{t \rightarrow \infty} x_{i}(t)-x_{j}(t)=0 \quad \forall i, j \forall \theta \in \Omega .
$$

In order to address this problem, we rewrite the uncertain multi-agent dynamical system (3) as

$$
\dot{x}(t)=-L(\theta) x(t)
$$

where $x=\left(x_{1}, \ldots, x_{n}\right) \in \mathbb{R}^{n}$ is the state vector, and $L(\theta)=$ $\left(L_{i j}(\theta)\right)_{n \times n}$ is the uncertain Laplacian matrix given by

$$
\begin{aligned}
& L_{i j}(\theta)=-G_{i j}(\theta) \forall i \neq j \\
& L_{i i}(\theta)=-\sum_{j=1, j \neq i}^{n} L_{i j}(\theta) .
\end{aligned}
$$

It is worth pointing out that the uncertain Laplacian matrix has the diffusion property that

$$
\sum_{j=1}^{n} L_{i j}(\theta)=0 \quad \forall i=1, \ldots, n .
$$

For robust second-order consensus problem, we consider the continuous-time uncertain multi-agent dynamical system described by

$$
\begin{aligned}
\dot{x}_{i}(t)= & \rho_{i}(t) \\
\dot{\rho}_{i}(t)= & \sum_{j=1, j \neq i}^{n} \alpha G_{i j}(\theta)\left(x_{j}(t)-x_{i}(t)\right) \\
& +\sum_{j=1, j \neq i}^{n} \beta G_{i j}(\theta)\left(\rho_{j}(t)-\rho_{i}(t)\right)
\end{aligned}
$$

where $x_{i} \in \mathbb{R}$ is the position state of the $i$-th node, $\rho_{i} \in \mathbb{R}$ is the velocity state of the $i$-th node, and $\alpha, \beta \in \mathbb{R}$ are constants. Different from first-order consensus, second-order consensus requires that not merely do the position states of agents tend to be the same, but also the velocity states of agent converge to a consistent value. Based on this we propose the problem of robust second-order consensus as follows.

Problem 2. To establish if, for any initial state, the uncertain multi-agent dynamical system (8) achieves robust secondorder consensus, i.e.

$$
\begin{aligned}
\lim _{t \rightarrow \infty} x_{i}(t)-x_{j}(t) & =0 \\
\lim _{t \rightarrow \infty} \rho_{i}(t)-\rho_{j}(t) & =0
\end{aligned} \quad \forall i, j \forall \theta \in \Omega .
$$

In order to address this problem, we rewrite the uncertain multi-agent dynamical system (8) as

$$
\begin{aligned}
& \dot{x}_{i}(t)=\rho_{i}(t) \\
& \dot{\rho}_{i}(t)=-\sum_{j=1}^{n} \alpha L_{i j}(\theta) x_{j}(t)-\sum_{j=1}^{n} \beta L_{i j}(\theta) \rho_{j}(t)
\end{aligned}
$$

where $x \in \mathbb{R}^{n}$ is the position state vector and $\rho \in \mathbb{R}^{n}$ is the velocity state vector. We define the global state vector as $y=\left(x^{\prime}, \rho^{\prime}\right)^{\prime} \in \mathbb{R}^{2 n}$. Then, system (10) can be rewritten in compact form as

$$
\dot{y}(t)=\widetilde{L}(\theta) y(t)
$$

where $\widetilde{L}(\theta)$ is the uncertain extended Laplacian matrix given by

$$
\widetilde{L}(\theta)=\left[\begin{array}{cc}
0 & I \\
-\alpha L(\theta) & -\beta L(\theta)
\end{array}\right] .
$$

\section{B. SOS Polynomials}

Let $f(\theta)$ be a polynomial of degree $2 m$ in $\theta \in \mathbb{R}^{r}$. Then, $f(\theta)$ can be always written as

$$
f(\theta)=\theta^{\{m\}^{\prime}}(F+C(\delta)) \theta^{\{m\}}
$$

where $\theta^{\{m\}}$ is a vector containing all monomials of degree less than or equal to $m$ in $\theta, F$ is a symmetric matrix, and $C(\delta)$ is a linear parametrization of the subspace

$$
\mathcal{C}=\left\{C=C^{\prime}: \theta^{\{m\}^{\prime}} C \theta^{\{m\}}=0\right\} .
$$


The representation (13) is known as Gram matrix method and square matrix representation (SMR). This representation allows one to establish whether a polynomial is SOS via LMIs. Indeed, $f(\theta)$ is SOS if there exist polynomials $f_{1}(\theta), f_{2}(\theta), \ldots$ such that

$$
f(\theta)=\sum_{i} f_{i}(\theta)^{2}
$$

and this condition holds if and only if there exists $\delta$ such that the following LMI feasibility test holds:

$$
F+C(\delta) \geq 0 \text {. }
$$

This technique can also be used in the case of matrix polynomials. Specifically, let $M(\theta)$ be a symmetric matrix polynomial of size $s \times s$ of degree $2 m$ in $\theta \in \mathbb{R}^{r}$ (this means that all the entries of $M(\theta)$ are polynomials whose highest degree in $\theta$ is $2 m$ ). Then, $M(\theta)$ can be written as

$$
M(\theta)=\Delta(\bar{M}+D(\delta), m, s)
$$

where

$$
\Delta(\bar{M}+D(\delta), m, s)=\left(\theta^{\{m\}} \otimes I\right)^{\prime}(\bar{M}+D(\delta))\left(\theta^{\{m\}} \otimes I\right),
$$

$I$ is the $s \times s$ identity matrix, $\bar{M}$ is a symmetric matrix, and $D(\delta)$ is a linear parametrization of the subspace

$$
\mathcal{D}=\left\{D=D^{\prime}: \Delta(D, m, s)=0\right\} .
$$

Similarly to the scalar case, $M(\theta)$ is SOS if there exist matrix polynomials $M_{1}(\theta), M_{2}(\theta), \ldots$ such that

$$
M(\theta)=\sum_{i} M_{i}(\theta)^{\prime} M_{i}(\theta)
$$

and this condition holds if and only if there exists $\delta$ such that the following LMI feasibility test holds:

$$
\bar{M}+D(\delta) \geq 0 .
$$

See for instance [16]-[18] and references therein for details and algorithms about SOS polynomials.

\section{CONDitions FOR Robust CONSEnsus}

In this section, the robust first-order and second-order consensus conditions are derived respectively.

\section{A. Robust First-Order Consensus}

Lyapunov stability theory is widely used to study the property of dynamical system. For the first time, we associate the robust consensus with Lyapunov stability theory, and we provide a new condition for investigating robust first-order consensus based on matrix inequalities. Specifically, define a matrix $V_{1} \in \mathbb{R}^{n \times n-1}$ such that

$$
\operatorname{img}\left(V_{1}\right)=\operatorname{ker}\left(1_{n}^{\prime}\right) \text {. }
$$

Then we get the transformed uncertain Laplacian matrix:

$$
\widehat{L}(\theta)=V_{1}^{\prime} L(\theta) V_{1} \text {. }
$$

Theorem 1: Robust first-order consensus for uncertain multi-agent system (with both positive and non-positive weighted digraph) can be achieved if and only if there exists a symmetric function $P_{1}: \mathbb{R}^{r} \rightarrow \mathbb{R}^{n-1 \times n-1}$ such that

$$
\left\{\begin{array}{l}
P_{1}(\theta)>0 \\
P_{1}(\theta) \widehat{L}(\theta)+\widehat{L}(\theta)^{\prime} P_{1}(\theta)>0
\end{array} \quad \forall \theta \in \Omega .\right.
$$

In order to investigate the condition of Theorem 1, we can exploit SOS matrix polynomials introduced in Section II-B. Indeed, it is easy to verify that (17) holds if there exist matrix polynomials $P_{1}(\theta), G_{1 i}(\theta)$ and a scalar $c>0$ such that

$$
\left\{\begin{array}{l}
G_{1 i}(\theta) \text { is SOS } \\
P_{1}(\theta)-I \text { is SOS } \\
R_{1}(\theta)-c I \text { is SOS }
\end{array}\right.
$$

where

$$
R_{1}(\theta)=P_{1}(\theta) \widehat{L}(\theta)+\widehat{L}(\theta)^{\prime} P_{1}(\theta)-\sum_{i=1}^{h} G_{1 i}(\theta) s_{1 i}(\theta) .
$$

In fact, whenever the constraints in (18) hold with $c>0$, for any $\theta \in \Omega$ it follows that $G_{1 i}(\theta) \geq 0, P_{1}(\theta)>0$ and

$$
\begin{aligned}
0 \leq & P_{1}(\theta) \widehat{L}(\theta)+\widehat{L}(\theta)^{\prime} P_{1}(\theta)-\sum_{i=1}^{h} G_{1 i}(\theta) s_{1 i}(\theta) \\
& -c I \\
\leq & P_{1}(\theta) \widehat{L}(\theta)+\widehat{L}(\theta)^{\prime} P_{1}(\theta)
\end{aligned}
$$

i.e. (17) holds.

The condition (18) can be formulated via a convex optimization problem by using the representation of matrix polynomials reported in Section II. Indeed, it directly follows that (17) holds if $c^{*}>0$, where $c^{*}$ is the solution of the convex optimization problem

$$
\begin{gathered}
c^{*}=\sup _{c, \bar{G}_{1 i}, \bar{P}_{1}, \delta} c \\
\text { s.t. }\left\{\begin{array}{l}
\bar{G}_{i 1} \geq 0 \\
\bar{F}_{1}+D_{1}(\delta)-c I-\sum_{i=1}^{h} \bar{U}_{1 i}\left(\bar{G}_{1 i}\right) \geq 0 \\
\operatorname{trace}\left(\bar{P}_{1}\right)=1 .
\end{array}\right.
\end{gathered}
$$

The matrices involved in this problem are defined by

$$
\begin{aligned}
G_{1 i}(\theta) & =\Delta\left(\bar{G}_{1 i}, m_{i}, n-1\right) \\
G_{1 i}(\theta) s_{1 i}(\theta) & =\Delta\left(\bar{U}_{1 i}\left(\bar{G}_{1 i}\right), m_{0}, n-1\right) \\
P_{1}(\theta) & =\Delta\left(\bar{P}_{1}, m, n-1\right) \\
R_{1}(\theta) & =\Delta\left(\bar{F}_{1}+D_{1}(\delta), m_{0}, n-1\right) .
\end{aligned}
$$

Here, $2 m_{i}$ is the degree of $G_{1 i}(\theta), 2 m$ is the degree of $P_{1}(\theta)$, and $2 m_{0}$ is the degree of $R_{1}(\theta)-c I$.

For an interaction topology with positive weighted interaction topology but without parametric uncertainties, it has been found that the topological structure determines whether the consensus can be achieved. The following theorem extends to the case of uncertain multi-agent dynamical systems three existing conditions found for the case of multi-agent dynamical systems without uncertainty [19], and provides a further condition in terms of zeros of a polynomial.

Theorem 2: For a given uncertain Laplacian matrix $L(\theta)$ in (6) and a network $\mathscr{G}=(\mathscr{A}, \mathscr{E}, G(\theta))$ with a positive weighted 
digraph, i.e. $\exists e_{i j} \in \mathscr{E}$ if and only if $G_{i j}(\theta)>0$, the following statements are equivalent.

a) Robust first-order consensus can be achieved.

b) $\forall \theta \in \Omega, L(\theta)$ has exactly one simple eigenvalue 0 and all the other eigenvalues have positive parts.

c) $\forall \theta \in \Omega$, the directed graph $\mathscr{G}$ has a spanning tree.

d) $\forall \theta \in \Omega, q(\theta) \neq 0$, where

$$
q(\theta)=\left.\frac{d}{d \lambda} l(\lambda, \theta)\right|_{\lambda=0}
$$

and

$$
l(\lambda, \theta)=\operatorname{det}(\lambda I-L(\theta)) .
$$

One way of checking the condition of Theorem 2 consists of using SOS polynomials and amounts to solving an LMI problem. Specifically, statement d) in Theorem 2 holds if there exist polynomials $g_{i}(\theta)$ and a scalar $c>0$ such that

$$
\left\{\begin{array}{l}
g_{i}(\theta) \text { is } \operatorname{SOS} \\
(-1)^{k} q(\theta)-c-\sum_{i=1}^{h} g_{i}(\theta) s_{2 i}(\theta) \text { is SOS }
\end{array}\right.
$$

where $k \in\{0,1\}$ is defined by

$$
k= \begin{cases}0 & \text { if } q\left(\theta_{0}\right)>0 \\ 1 & \text { otherwise }\end{cases}
$$

and $\theta_{0}$ is any vector $\theta$ in $\Omega$ which can be freely chosen.

\section{B. Robust Second-Order Consensus}

Let us consider the problem of establishing robust secondorder consensus. For this problem, we exploit the uncertain expanded Laplacian matrix $\widetilde{L}(\theta)$. Extending the results given in [12] for the case of multi-agent dynamical systems without uncertainty, one has that robust second-order consensus for the uncertain multi-agent dynamical system (11) can be obtained if and only if $-\widetilde{L}(\theta)$ has only one zero eigenvalue of algebraic multiplicity two and all the other eigenvalues are in the open right half plane.

Starting from this result, we provide a new condition for investigating robust second-order consensus based on matrix inequalities. Specifically, define vectors as

$$
u_{1}=\left(\begin{array}{c}
1_{n} \\
0_{n}
\end{array}\right), \quad u_{2}=\left(\begin{array}{c}
0_{n-1} \\
1_{n}
\end{array}\right) .
$$

Let $V_{2} \in \mathbb{R}^{2 n \times 2 n-1}$ and $V_{3} \in \mathbb{R}^{2 n-1 \times 2 n-2}$ be matrices such that

$$
\begin{aligned}
& \operatorname{img}\left(V_{2}\right)=\operatorname{ker}\left(u_{1}^{\prime}\right) \\
& \operatorname{img}\left(V_{3}\right)=\operatorname{ker}\left(u_{2}^{\prime}\right) .
\end{aligned}
$$

Let us define the transformed uncertain expanded Laplacian matrix:

$$
\breve{L}(\theta)=-V_{3}^{\prime} V_{2}^{\prime} \widetilde{L}(\theta) V_{2} V_{3} .
$$

Theorem 3: Robust second-order consensus for uncertain multi-agent system with both positive and non-positive weighted digraph can be achieved if and only if there exists a symmetric function $P_{2}: \mathbb{R}^{r} \rightarrow \mathbb{R}^{2 n-2 \times 2 n-2}$ such that

$$
\left\{\begin{array}{l}
P_{2}(\theta)>0 \\
P_{2}(\theta) \breve{L}(\theta)+\breve{L}(\theta)^{\prime} P_{2}(\theta)>0
\end{array} \quad \forall \theta \in \Omega .\right.
$$

In order to investigate the existence of a function $P_{2}(\theta)$ satisfying condition (27), we can exploit SOS matrix polynomials. It is easy to verify that (27) holds if there exist matrix polynomials $P_{2}(\theta), G_{3 i}(\theta)$ and a scalar $c>0$ such that

$$
\left\{\begin{array}{l}
G_{3 i}(\theta) \text { is SOS } \\
P_{2}(\theta)-I \text { is SOS } \\
R_{2}(\theta)-c I \text { is SOS }
\end{array}\right.
$$

where

$$
R_{2}(\theta)=P_{2}(\theta) \breve{L}(\theta)+\breve{L}(\theta)^{\prime} P_{2}(\theta)-\sum_{i=1}^{h} G_{3 i}(\theta) s_{3 i}(\theta) .
$$

Before concluding this section, let us remark that the proposed results for establishing robust consensus in uncertain multiagent systems require the solution of optimization problems, in contrast to existing conditions for establishing consensus in uncertainty-free multi-agent systems where one just needs to check the eigenvalues of the Laplacian matrices. Unfortunately, this is unavoidable, as it happens also for the simpler problem of establishing robust stability of uncertain linear systems, see for instance [17].

\section{NUMERICAL EXAMPLES}

This section presents some illustrative examples where robust first-order and second-order consensus are investigated for uncertain multi-agent dynamical systems. The optimization problems are solved with the standard Matlab toolbox SeDuMi. The SMR matrices are built using the algorithms reported in [17] and references therein.

\section{A. Example 1}

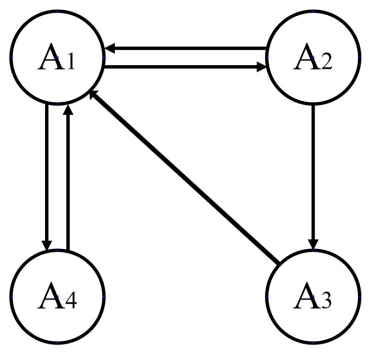

Fig. 1: Digraph of a four-agent system

In this example we consider the uncertain four-agent system shown in Figure 1. It is assumed that the network is affected by an uncertain parameter, specifically

$$
G(\theta)=\left[\begin{array}{cccc}
1 & 2-2 \theta & 5+\theta & 2+\theta \\
3 \theta & 1 & 0 & 0 \\
0 & 4-3 \theta & 1 & 0 \\
2+3 \theta & 0 & 0 & 1
\end{array}\right]
$$

where $\theta$ is constrained in the set $\Omega$ chosen as $\Omega=[0,1]$. Hence, we have $n=4$ and $r=1$. Moreover, $\Omega$ can be described as in (2) with

$$
s_{1}(\theta)=\theta(1-\theta) .
$$


According to (6), the Laplacian matrix $L(\theta)$ is given by:

$$
L(\theta)=\left[\begin{array}{cccc}
9 & -2+2 \theta & -5-\theta & -2-\theta \\
-3 \theta & 3 \theta & 0 & 0 \\
0 & 4-3 \theta & 4-3 \theta & 0 \\
-2-3 \theta & 0 & 0 & 2+3 \theta
\end{array}\right]
$$

We observe that $G(\theta)$ is positive since all its entries are nonnegative for all $\theta \in \Omega$. This implies that we can use either condition (17) or statement d) of Theorem 2 to investigate robust first-order consensus.

First, we use condition (17) by looking for a constant matrix function $P_{1}(\theta)$. By solving (20) we can find $c^{*}=0.9792$. Therefore, robust first-order consensus is achieved.

Then, let us use statement d) of Theorem 2. In particular, the polynomial $q(\theta)$ is given by

$$
q(\theta)=18 \theta^{3}+6 \theta^{2}-112 \theta-56 .
$$

According to statement d) of Theorem 2, robust first-order consensus is achieved if and only if $q(\theta) \neq 0$ for all $\theta \in[0,1]$. In this case, it is easy to see that $q(\theta)$ satisfies this property since $q(\theta)$ is an univariate polynomial with roots $2.79,-1.3316$ and -1.7917 which are all lying outside $[0,1]$. Nevertheless, let us use condition (23). In this case $k=1$ and by simply choosing a multiplier $g_{1}(\theta)$ of degree 2 we find that this condition holds with $c=56$, which proves that statement d) of Theorem 2 is satisfied. Figure 2 shows the process of robust first-order consensus with the initial states and $\theta$ randomly chosen in $[0,1]$ for five times.

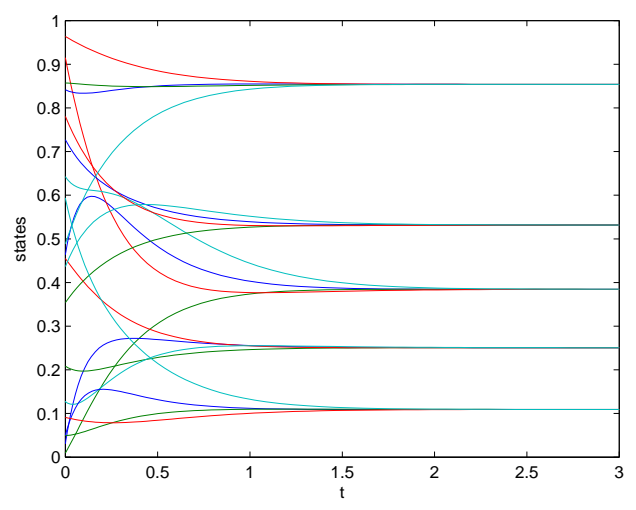

Fig. 2: Trajectories of robust first-order consensus

Next, let us consider the problem of establishing whether this uncertain network is able to achieve robust second-order consensus with $\alpha=\beta=1$ in the system (8), and we look for a constant matrix function $P_{2}(\theta)$ satisfying (27). Nevertheless, let us use the condition (28), and we can find $c^{*}=0.0913$. Therefore, robust second-order consensus is achieved with chosen $\alpha$ and $\beta$. In this case, the uncertain extended Laplacian matrix is given by

$$
\left[\begin{array}{cccccccc}
0 & 0 & 0 & 0 & 1 & 0 & 0 & 0 \\
0 & 0 & 0 & 0 & 0 & 1 & 0 & 0 \\
0 & 0 & 0 & 0 & 0 & 0 & 1 & 0 \\
0 & 0 & 0 & 0 & 0 & 0 & 0 & 1 \\
9 & l_{1} & l_{2} & l_{3} & -9 & l_{1} & l_{2} & l_{3} \\
l_{4} & -l_{4} & 0 & 0 & l_{4} & -l_{4} & 0 & 0 \\
0 & l_{5} & -l_{5} & 0 & 0 & l_{5} & -l_{5} & 0 \\
l_{6} & 0 & 0 & -l_{6} & l_{6} & 0 & 0 & -l_{6}
\end{array}\right]
$$

where $l_{1}=2-2 \theta, l_{2}=5+\theta, l_{3}=2+\theta, l_{4}=3 \theta, l_{5}=4-3 \theta$, $l_{6}=2+3 \theta$.

\section{B. Example 2}

In this example we consider the uncertain matrix $G(\theta)$ given by

$$
G(\theta)=\left[\begin{array}{cccc}
1 & 5+2 \theta_{1} \theta_{2} & 2-3 \theta_{1}^{2} & -3 \theta_{1} \theta_{2} \\
2 \theta_{2}^{2}-3 \theta_{1} \theta_{2} & 1 & 0 & 0 \\
0 & 4+2 \theta_{1}^{2} & 1 & 0 \\
3 \theta_{1} \theta_{2}+6 & 0 & 0 & 1
\end{array}\right]
$$

where $\theta \in \mathbb{R}^{2}$ is constrained in the set $\Omega$ chosen as $\Omega=$ $[-1,1]^{2}$. Hence, we have $n=4$ and $r=2$. Moreover, $\Omega$ can be described as in (2) with

$$
s_{i}(\theta)=1-\theta_{i}^{2} \quad \forall i=1,2 .
$$

In this case $G(\theta)$ is not positive, hence let us use condition (17) to investigate robust first-order consensus. We look for a constant matrix function $P_{1}(\theta)$ satisfying (17), and by solving (20) we find $c^{*}=0.769$. Therefore, robust first-order consensus is achieved.

Next, let us consider the problem of establishing whether this uncertain network is able to achieve robust second-order consensus with $\alpha=1, \beta=0.25$ in the system (8). We look for a constant matrix function $P_{2}(\theta)$ satisfying (27). Let us use the condition (28), and we find $c^{*}=-0.0024$, which does not prove (28). We repeat the procedure by looking for a matrix function $P_{2}(\theta)$ of degree 2 , and we find a positive $c^{*}$. Therefore, robust second-order consensus is achieved.

\section{Example 3}

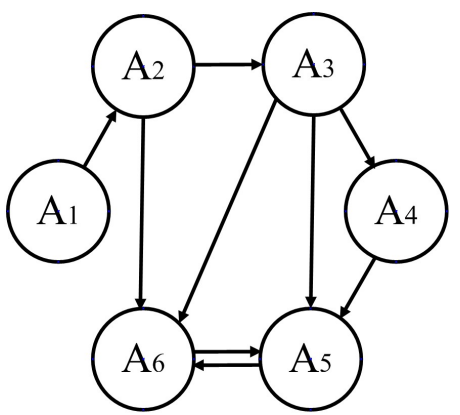

Fig. 3: Digraph of a six-agent system

With a topology shown in Figure 3, an uncertain six-agent system is considered in this example. It is assumed that the 
network is affected by two uncertain parameters, i.e. $\theta_{1}$ and $\theta_{2}$. Specifically the uncertain matrix $G(\theta)$ is given by

$$
\left[\begin{array}{cccccc}
1 & 0 & 0 & 0 & 0 & 0 \\
3+2 \theta_{1} & 1 & 0 & 0 & 0 & 0 \\
0 & 3-\theta_{2} & 1 & 0 & 2 \theta_{1}+\theta_{2} & 0 \\
0 & 0 & 5+2 \theta_{1} & 1 & 0 & 0 \\
0 & 0 & 0 & 5 & 1 & 3-4 \theta_{2} \\
0 & 5 & 2-3 \theta_{1} & 0 & 2-\theta_{2} & 1
\end{array}\right] .
$$

where $\theta \in \mathbb{R}^{2}$ is constrained in the set $\Omega$ chosen as $\Omega=\{\theta$ : $\|\theta\| \leq 1\}$. Hence, we have $n=4$ and $r=2$. Moreover, $\Omega$ can be described as in (2) with

$$
s_{1}(\theta)=1-\theta_{1}^{2}-\theta_{2}^{2} .
$$

Also in this case $G(\theta)$ is not positive, hence let us use condition (17) to investigate robust first-order consensus. We look for a constant matrix function $P_{1}(\theta)$ satisfying (17), and by solving (20) we find $c^{*}=0.1135$, i.e. robust first-order consensus is achieved.

Next, let us consider the problem of establishing whether this uncertain network is able to achieve robust second-order consensus with $\alpha=1, \beta=0.6$ in the system (8). We look for a constant matrix function $P_{2}(\theta)$ satisfying (27). Let us use the condition (28), and we find $c^{*}<0$, which does not prove (28). We repeat the procedure by looking for a matrix function $P_{2}(\theta)$ of degree 1 , and we find $c^{*}=0.0034$, i.e. robust second-order consensus is achieved.

\section{CONCLUSIONS}

In this paper we have addressed robust first-order consensus and robust second-order consensus for a class of uncertain multi-agent dynamical systems. Specifically, we have considered a generic framework where the system is described by a weighted adjacency matrix whose entries are polynomial functions of an uncertain vector constrained in a semialgebraic set. For this uncertain topology, we have provided necessary and sufficient conditions for ensuring robust consensus in both cases of positive and non-positive weighted adjacency matrices. Moreover, we have shown how these conditions can be easily investigated through convex programming by using standard software. Various future directions can be taken starting from the results proposed in this paper, for instance one can consider switching topology adopting the frameworks introduced in [20], [21] and LMI techniques for switching systems as the one introduced in [22]. Also, multiagent dynamical systems with rational dependence on the uncertainty and/or time-varying uncertainty can be considered adopting the methodology proposed in [23].

\section{APPENDIX}

\section{A. Proof of Theorem 1}

We observe that $1_{n}$ is an eigenvector of $L(\theta)$ corresponding to the eigenvalue zero. Moreover, observe that $V_{1}^{\prime} L(\theta) V_{1}$ has the same eigenvalues of $L(\theta)$ except that the algebraic multiplicity of the eigenvalue zero has been decreased of one. i.e.

$$
\operatorname{spc}(\widehat{L}(\theta)) \cup\{0\}=\operatorname{spc}(L(\theta))
$$

Let us define a dynamical system

$$
\dot{\hat{x}}(t)=-\widehat{L}(\theta) \hat{x}(t) \text {. }
$$

We observe that $x=\gamma 1_{n}$ is the equilibrium point of (30), $\forall \gamma \in$ $\mathbb{R}$. Hence the robust first-order consensus can be achieved is equivalent to the statement that (30) is asymptotically stable. According to (29) and the Lyapunov stability theorem, (30) is asymptotically stable for all $\theta \in \Omega$ if and only if $L(\theta)$ has exactly one simple eigenvalue 0 and all the other eigenvalues have positive parts. From Lyapunov stability theorem for linear systems, this is equivalent to say that there exists $P_{1}(\theta)$ such that (17) holds for all $\theta \in \Omega$. Therefore, the theorem holds.

\section{B. Proof of Theorem 2}

Assume the Laplacian matrix $L(\theta)$ is constructed by (6). Then, the first three statements are equivalent and follow directly from the analogous ones found for the case of multiagent dynamical systems without uncertainty [19]. From Lemma 3.3 in [19], one has that $\Re\left(\lambda_{i}(L(\theta))\right) \geq 0, \forall i=1,2 \ldots, n$, $\forall \theta \in \Omega$. Moreover, statement d) implies that $L(\theta)$ has exactly one zero eigenvalue, $\forall \theta \in \Omega$. Thus, statements b) and d) are equivalent. Therefore, the theorem holds.

\section{Proof of Theorem 3}

Observe that $u_{1}$ is an eigenvector of $\widetilde{L}(\theta)$ corresponding to the eigenvalue zero. Moreover, observe that $V_{2}^{\prime} \widetilde{L}(\theta) V_{2}$ has the same eigenvalues of $\widetilde{L}(\theta)$ except that the algebraic multiplicity of the eigenvalue zero has been decreased of one. Similarly, it follows that $V_{3}^{\prime} V_{2}^{\prime} \widetilde{L}(\theta) V_{2} V_{3}$ has the same eigenvalues of $\widetilde{L}(\theta)$ except that the algebraic multiplicity of the eigenvalue zero has been decreased of two. Hence, it follows that robust second-order consensus can be achieved if and only if $-\widetilde{L}(\theta)$ has all the eigenvalues in the open right half plane for all $\theta \in \Omega$. From Lyapunov stability theorem for linear systems, this is equivalent to say that there exists $P_{2}(\theta)$ such that (27) holds for all $\theta \in \Omega$. Therefore, the theorem holds.

\section{REFERENCES}

[1] N. J. Cotton and B. M. Wilamowski. Compensation of nonlinearities using neural networks implemented on inexpensive microcontrollers. IEEE Transactions on Industrial Electronics, 58(3):733-740, 2011.

[2] B. Shen, Z. Wang, Y. S. Hung, and G. Chesi. Distributed H-infinity filtering for polynomial nonlinear stochastic systems in sensor networks. IEEE Transactions on Industrial Electronics, 58(5):1971-1979, 2011.

[3] D. Hunter, H. Yu, M.S. Pukish, and J. Kolbusz. Selection of proper neural network sizes and architecturesa comparative study. IEEE Transactions on Industrial Informatics, 8(2):228-240, 2012.

[4] H. Guo, K. S. Low, and H. A. Nguyen. Optimizing the localization of a wireless sensor network in real time based on a low-cost microcontroller. IEEE Transactions on Industrial Electronics, 58(3):741-749, 2011.

[5] D. Bruckner, C. Picus, R. Velik, W. Herzner, and G. Zucker. Hierarchical semantic processing architecture for smart sensors in surveillance networks. IEEE Transactions on Industrial Informatics, 8(2):291-301, 2012.

[6] J. Ploennigs, V. Vasyutynskyy, and K. Kabitzsch. Comparative study of energy-efcient sampling approaches for wireless control networks. IEEE Transactions on Industrial Informatics, 6(3):416-424, 2010. 
[7] C. Huang, Y. Bai, and X. Liu. H-innity state feedback control for a class of networked cascade control systems with uncertain delay. IEEE Transactions on Industrial Informatics, 6(1):62-72, 2010.

[8] Y. B. Zhao, J. Kim, and G. P. Liu. Error bounded sensing for packetbased networked control systems. IEEE Transactions on Industrial Electronics, 58(5):1980-1989, 2011.

[9] P. Mart, A. Camacho, M. Velasco, and M. E. M. B. Gaid. Runtime allocation of optional control jobs to a set of can-based networked control systems. IEEE Transactions on Industrial Informatics, 6(4):503520,2010

[10] Alphan Ulusoy, Ozgur Gurbuz, and Ahmet Onat. Wireless model-based predictive networked control system over cooperative wireless network. IEEE Transactions on Industrial Informatics, 7(1):41-51, 2011.

[11] Ahmet Onat, Teoman Naskali, Emrah Parlakay, and Ozan Mutluer. Control over imperfect networks: Model-based predictive networked contro systems. IEEE Transactions on Industrial Electronics, 58(3):905-913, 2011.

[12] W. Yu, G. Chen, and M. Cao. Some necessary and sufficient conditions for second-order consensus in multi-agent dynamical systems. Automatica, 46(6):1089-1095, 2010.

[13] R.J. Bril and J.J. Lukkien. Transparent synchronization protocols for compositional real-time systems. IEEE Transactions on Industrial Informatics, 8(2):322-336, 2012.

[14] J.A. Suul, A. Luna, P. Rodriguez, and T. Undeland. Voltage-sensor-less synchronization to unbalanced grids by frequency-adaptive virtual flux estimation. IEEE Transactions on Industrial Electronics, 59(7):2910 2923, 2012.

[15] Z. An, H. Zhu, X. Li, C. Xu, Y. Xu, and X. Li. Voltage-sensor-less synchronization to unbalanced grids by frequency-adaptive virtual flux estimation. IEEE Transactions on Industrial Electronics, 58(6):2205$2215,2011$.

[16] G. Chesi. On the gap between positive polynomials and sos of polynomials. IEEE Transactions on Automatic Control, 52(6):10661072, 2007.

[17] G. Chesi, A. Garulli, A. Tesi, and A. Viccino, editors. Homogeneous Polynomial Forms for Robustness Analysis of Uncertain Systems. Springer-Verlag, 2009.

[18] G. Chesi. LMI techniques for optimization over polynomials in control: a survey. IEEE Transactions on Automatic Control, 55(11):2500-2510, 2010.

[19] W. Ren and R.W. Beard. Consensus seeking in multiagent systems under dynamically changing interaction topologies. IEEE Transactions on Automatic Control, 50(5):655-661, 2005.

[20] L. Zhang, P. Shi, E. Boukas, and C. Wang. H-infinity control of switched linear discrete-time systems with polytopic uncertainties. optimal control. Optimal Control, Applications and Methods, 27(5):273-291, 2006.

[21] L. Zhang, P. Shi, C. Wang, and H. Gao. Robust h-infinity filtering for switched linear discrete-time systems with polytopic uncertainties. International Journal of Adaptive Control and Signal Processing, 20(6):291304, 2006.

[22] G. Chesi, P. Colaneri, J. C. Geromel, R. H. Middleton, and R. Shorten A nonconservative lmi condition for stability of switched systems with guaranteed dwell time. IEEE Transactions on Automatic Control, 57(5):1297-1302, 2012.

[23] G. Chesi. Robust stability of time-varying uncertain systems with rational dependence on the uncertainty. IEEE Transactions on Automatic Control, 55(4):2353-2357, 2010.

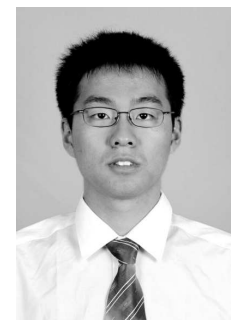

Dongkun Han received the B.S. degree in Electrical Engineering from the South China Agriculture University, Guangzhou, China, in 2007. He is currently working toward the Ph.D. degree in control systems, Department of Electrical and Electronic Engineering, University of Hong Kong, Hong Kong. His research interests include robust control, optimization control and cooperative control of multi-agent systems.
Graziano Chesi received the Laurea in Information Engineering from the University of Florence in 1997 and the Ph.D. in Systems Engineering from the University of Bologna in 2001. He was with the Department of Information Engineering of the University of Siena during 2000-2006 and then he joined the Department of Electrical and Electronic Engineering of the University of Hong Kong. Dr. Chesi has served as Associate Editor for Automatica, BMC Research Notes, the European Journal of Control, the IEEE Transactions on Automatic Control, and Systems \& Control Letters. Also, he has served as Guest Editor of the Special Issues on Positive Polynomials in Control, Systems Biology, and Visual Servoing for various journals. He is the Founder and Chair of the Technical Committee on Systems with Uncertainty of the IEEE Control Systems Society. He is author of the book "Homogeneous Polynomial Forms for Robustness Analysis of Uncertain Systems" (Springer, 2009) and editor of the book "Visual Servoing via Advanced Numerical Methods" (Springer, 2010). He is first author in more than 100 technical publications.

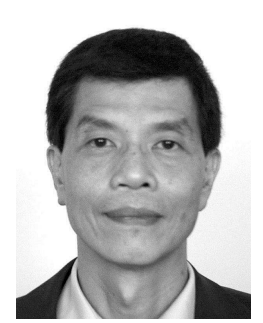

Yeung Sam Hung received his B.Sc. (Eng) in Electrical Engineering and B.Sc. in Mathematics, both from the University of Hong Kong, and his M.Phil. and Ph.D. degrees from the University of Cambridge. He has worked at the University of Cambridge and the University of Surrey before he joined the University of Hong Kong, where he is currently a professor at the Department of Electrical and Electronic Engineering. He has authored and coauthored over 200 publications in books, journals and conference proceedings. He is a fellow of the Hong Kong Institution of Engineers and the Institution of Engineering and Technology, UK. His research interests include control systems, computer vision and biomedical engineering. 